\title{
ESTUDIO CITOPATOLOGICO DE LAS LESIONES PRE-MALIGNAS Y MALIGNAS DEL CUELLO UTERINO
}

\author{
ST. LUKE'S HOSPITAL CENTER. - WOMAN'S HOSPITAL. - New York City
}

Dr. Miguel A. Pulido, M.D.

El presente es un estudio corto pero completo de los frotis Papanicolaou anormales y su relación con las diferentes lesiones cervicales, durante los años 1966 y 1967 en el St. Luke's Hospital Center, Woman's Hospital, de New York.

La correlación de la citología con la patología, y el diagnóstico de las lesiones cervicales hecho según los diferentes procedimientos quirúrgicos, diagnósticos o terapéuticos, es el objeto del presente trabajo. Se presenta un análisis de las 3 principales lesiones cervicales: Displasia, Carcinoma In-Situ y Carcinoma Invasi- vo. El material se obtuvo del comité de Tumores y del departamento de citología.

Tabla № 1. Representa el número total de frotis citológicos durante los años 1966 y 1967: 19.135, de éstos $16.683(87,1 \%)$ representan los frotis ginecológicos, con un total de 186 $(1,13 \%)$ clasificados como "anormales", entendiéndose por anormales aquellos frotis clases III, IV y V. Nuestra incidencia es similar a la presentada por S. Piver y colaboradores, quienes reportaron 17.487 frotis cérvico-vaginales de los cuales 236 $(1,3 \%)$ fueron positivos.

TABLA N: 1

FROTIS PAPANICOLAOU (1966-1967)

St. Luke's Hospital Center, New York

\begin{tabular}{lrrrr}
\hline & 1966 & 1967 & Total & $\%$ \\
\hline No total de Frotis & 8.865 & 10.270 & 19.135 \\
Frotis Ginecológicos & 7.921 & 8.762 & 16.683 & 87,1 \\
Frotis Ginecológicos Anormales* & 75 & 111 & 186 & 1,13 \\
* Clases III - IV - V. & & & & \\
\hline
\end{tabular}

Tabla No 2. Distribución de 186 frotis Papanicolaou en las diferentes lesiones cervicales: 2 fueron clase III con un diagnóstico histológico negativo, las biopsias cervicales mostraron epitelio cervical normal y el material obtenido en el curetaje fue inadecuado para un diagnóstico satisfactorio; posteriormente el frotis fue reportado normal sin más tratamiento quirúrgico. 38 frotis no tuvieron diagnóstico histológico: la mayoría 
TABLA NN 2

LESIONES CERVICALES

\begin{tabular}{|c|c|c|c|c|}
\hline & Caridad & Privados & & Total \\
\hline Negativos & 2 & & 2 & \\
\hline Sin Diagnóstico Histológico & 32 & 6 & 38 & \\
\hline Cervicitis & 8 & 1 & 9 & $(4,8 \%)$ \\
\hline Displasias & 28 & 3 & 31 & $(16,1 \%)$ \\
\hline Carcinoma In-situ & 33 & 25 & 58 & $(31,2 \%)^{*}$ \\
\hline Carcinoma Invasivo & 28 & 20 & 48 & $(25,8 \%)$ \\
\hline TOTAL & 131 & 55 & 186 & \\
\hline * Incluyendo 5 microinvasivos. & & & & \\
\hline
\end{tabular}

clase III, asociados con infección tricomonial, los cuales después de tratamiento adecuado regresaron a la normalidad. 9 casos $(4,8 \%)$ de Cervicitis: con frotis clase III y tricomonas. 31 casos $(16,1 \%)$ representan diferentes grados de displasias: de mínima a severa. 58 casos $(31,2 \%)$ fueron de Carcinoma In-Situ, incluyendo 5 casos de carcinoma microinvasivo. 48 casos $(25,8 \%)$ mostraron carcinoma invasivo. Hubo un total de 131 pacientes de caridad y 55 privados.

TABLA № 3

DistribuCION DE LAS Lesiones CERVICALES SEgun LA EDAD

\begin{tabular}{lcrrrrr}
\hline & $\mathbf{2 0}$ & $\mathbf{2 0 - 2 9}$ & $\mathbf{3 0 - 3 9}$ & $\mathbf{4 0 - 4 9}$ & $\mathbf{5 0}$ & Total \\
\hline Cervicitis & 1 & 1 & 5 & 2 & & 9 \\
Displasia & & 10 & 15 & 4 & 2 & 31 \\
Carcinoma In-situ & & 10 & 22 & 20 & 6 & 58 \\
Carcinoma Invasivo & & 2 & 3 & 11 & 32 & $\mathbf{4 8}$ \\
$\quad$ TOTAL & 1 & 23 & 45 & 37 & 40 & 146 \\
\hline
\end{tabular}

Tabla No 3. Muestra la distribución de las lesiones cervicales según la edad: 1 caso en una paciente menor de 20 años, con cervicitis y frotis clase III. Cervicitis con frotis anormales fue más común entre los 30 y 39 años de edad. Las displasias fueron más comunes en la cuarta década (15 casos); 10 casos se encontraron entre los 20 y 29 años de edad; 4 casos en la quinta década y 2 por encima de los 50 años de edad. Así, la incidencia más alta fue entre los 20 y 40 años, con un promedio similar a las figuras dadas por Richart: 34,5 .
Carcinoma in-situ se encontró casi co nigual frecuencia entre los 30 y 39 años, y 40-49 años de edad; Kistner da una edad promedio de 38; Parsons \& Sommers: 30 a 40 años. J. Ferguson y colaboradores reportó una alta incidencia de carcinoma insitu en la tercera década de la vida (20-29), pero hay que tener en cuenta que en su material hubo también una alta incidencia de matrimonios jóvenes, relaciones sexuales a muy temprana edad y bajo estado socioeconómico. Hubo 6 casos de Carcinoma in-situ en la sexta década, en nuestro grupo. 
Vol. $X X$

NNo 5 ESTUdIO CITOPATOLOGICO DE LESIONES PRE- Y MALIGNAS DEL CUELLO UTERINO

Carcinoma Invasivo: La incidencia más alta fue entre los 50 y 60 años de edad; Kistner da una edad promedio de 48. Hubo 5 carcinomas microinvasivos entre los 34 y 54 años de edad.

En resumen, el gran número de lesiones cervicales se encontró en el grupo de 30 a 39 años de edad, con prelación de carcinoma in-situ, se- guido por el grupo de los 50 años o más de edad, principalmente representado por carcinoma invasivo; en tercer lugar el grupo de los 40-49 años con prelación de nuevo de carcinoma in-situ y finalmente el grupo más joven: 20-29 años de edad con número igual de displasias y carcinoma in-situ, pero con menos frecuencia que en los otros grupos.

TABLA No 4

DISTRIBUCION DE LAS LESIONES CERVICALES SEGUN PARIDAD Y EMBARAZO

\begin{tabular}{lccc}
\hline & Nulíparas & Multíparas & Embarazadas \\
\hline Cervicitis & 3 & 6 & 5 \\
Displasia & 6 & 25 & 2 \\
Carcinoma In-situ & 9 & 49 & 7 \\
Carcinoma Invasivo & 9 & 39 & 7 \\
TOTAL & 27 & 119 & \\
\hline
\end{tabular}

Tabla № 4. Lesiones Cervicales distribuídas de acuerdo con Paridad y Embarazo. La mayoría de las lesiones cervicales fueron encontradas en multíparas ( 1 o más hijos); 9 casos de carcinoma in-situ y 9 de carcinoma invasivo fueron encontrados en nulíparas. Ferguson y colaboradores reportaron 103 casos de carcinoma in-situ, 14 de los cuales fueron en nulíparas.

5 pacientes mostraron displasia durante el embarazo: la primera fue tratada con conización en el primer trimestre, el parto se presentó en otro hospital y el control se perdió; a la segunda paciente se le practicó una biopsia que dio severa displasia, ésta paciente tuvo varios frotis clase III antes de la biopsia y uno más antes del parto, estaba en control en el momento de escribirse este trabajo. En la tercera paciente el diagnóstico se hizo por biopsia, un dispositivo intra-uterino fue insertado durante el puerperio, posteriormente el frotis cervical fue de nuevo clase III, se practicó un curetaje y múltiples biopsias que resultaron negativas; la cuarta paciente mostró displasia en una biopsia, 6 semanas post-parto se practicó una conización la cual también mostró displasia, después de la conización se puso bajo contraceptivos orales, se siguió por 9 meses y se perdió el control; en la quinta paciente el diagnóstico también se hizo por biopsia, después del parto el frotis fue clase II-R y luego III, múltiples biopsias cervicales mostraron cervicitis, ésta paciente está bajo control en el presente momento.

Dos pacientes embarazadas, con Carcinoma in-situ: la primera tuvo un frotis clase IV, se practicó una conización a las 8 semanas, el parto se presentó a término, 13 meses después de la conización se practicó una histerectomía total abdominal por fibromas, y no se encontró lesión residual, los frotis después del parto fueron negativos. La segunda paciente tuvo un frotis clase III (leído fuera del hospital), se practicó coniza- 
TABLA NN 5

CONTRACEPTIVOS $Y$ LESIONES CERVICALES

\begin{tabular}{|c|c|c|c|c|c|c|c|c|c|c|}
\hline & III & $\begin{array}{l}\text { Oral } \\
\text { IV V }\end{array}$ & SF. & III & $\begin{array}{l}\text { DIU. } \\
\text { IV V SF. }\end{array}$ & $\begin{array}{l}\text { Lig. Tron } \\
\text { III IV V }\end{array}$ & $\begin{array}{l}\text { mpas } \\
\text { SF. }\end{array}$ & III & $\begin{array}{l}\text { Otros } \\
\text { IV V SF. }\end{array}$ & Total \\
\hline Displasia & 3 & 1 & & 1 & & & & & & 5 \\
\hline Carcinoma In-situ & 1 & 3 & 2 & & 2 & 2 & & 1 & & 11 \\
\hline Carcinoma Invasivo & & 1 & & & & & 1 & & 1 & 3 \\
\hline Sin Dx. Histológico & 4 & & & 1 & & & & & & 5 \\
\hline
\end{tabular}

ción a las 16 semanas y 3 días más tarde abortó, la paciente está bajo control privado.

Tabla No 5. Incidencia de Lesiones Cervicales en Pacientes Usando Contraceptivos: los métodos más usados fueron en su mayoría medicamentos orales, (Enovid, Ortho-novum y Cquens), algunas pacientes usaron el dispositivo intra-uterino, otras usaron diafragma y unas pocas se sometieron a esterilización permanente (Ligadura y Sección de las trompas). La mayoría de las lesiones cervicales se encontraron en pacientes bajo contraceptivos orales: 4 displasias 6 carcinomas in-situ y 1 carcinoma invasivo; las tabletas habían sido usadas por un período de 6 meses a 3 años; hubo 4 pacientes con frotis clase III pero en las cuales no se efectuó diagnóstico histológico. Dos pacientes con Carcinoma in-situ y una con displasia quienes estaban usando el dispositivo intrauterino; en una paciente no se hizo diagnóstico histológico. Después de esterilización permanente ( 1 a 3 años) 2 pacientes desarrollaron carcinoma in-situ y 1 carcinoma invasivo. $Y$ finalmente 2 pacientes con diafragma: una desarrolló carcinoma in-situ y la otra invasivo.

En conclusión hubo 5 displasias, 10 carcinomas in-situ y 3 carcinomas invasivos, 5 pacientes con frotis anormales y sin diagnóstico histológico.
Hiperplasia de las glándulas endocervicales, semejando adenocarcinoma, ha sido encontrada en pacientes usando contraceptivos (en la Tabla No 13 se discute un caso y se presentan microfotografías), los casos aquí presentados fueron todos lesiones del epitelio escamoso. S. Piver \& R. Bolognese estudiaron 692 frotis cérvicovaginales en pacientes usando dispositivos intra-uterinos y encontraron solamente 3 displasias cervicales. Fuchs no encontró lesiones cervicales premalignas o malignas en 751 pacientes usando dispositivos.

Uno de los más recientes trabajos sobre el efecto de los contraceptivos orales en las lesiones pre-malignas del cuello uterino es aquel de J. Arsenault, J. E. Ayre y colaboradores, del National Cancer Cytology Center en New York: estos investigadores estudiaron el efecto de Enovid (Norethynodrel \& Mestranol) en pacientes en quienes se sabía tenían displasias cervicales y carcinoma in-situ, ellos observaron un marcado $y$ consistente aumento en exfoliación de células displásticas y atípicas, además del esperado aumento en la cornificación inducida por los estrógenos. También fue observado que la administración de estrógenos no produjo la transformación de Carcinoma in-situ en carcinoma invasivo; la acción hormonal directa es reversible cuando la hormona es descontinuada, pero no al estado normal sino 
al estado inmediatamente anterior al comienzo del uso de los contraceptivos. La dosis usada fue de 2-5-10 mg./d. por 20 días, aunque también se usó medicación continua, y los pacientes fueron observados con frotis citológicos regulares hasta 3 y 4 años. En 60 mujeres con displasia del cuello uterino, ninguna de ellas mostró progresión acelerada después de 3 años de terapia con Enovid 6 desarrollaron "de novo" carcinoma insitu, éstos fueron considerados accidentales debido a que la administración oral de progestógenos databa solamente de unos meses atrás, y la lesión indudablemente estaba presente varios años antes de la administración de Enovid. Los autores de este trabajo establecieron que las lesiones pueden permanecer dormidas por varios años, durante la administración de Enovid, antes de que ellas progresen a un proceso más anaplástico.

En resumen, en el presente momento no hay evidencia de que los progestógenos orales influyan en la transformación de las lesiones cervicales a un proceso pre-maligno o maligno. Un número considerable de pacientes deberá observarse por lo menos durante 5 a 10 años para así poder llegar a una conclusión definitiva.

(Microfotografías de frotis Papanicolaou; I a V).

TABLA № 6

FROTIS PAPANICOLAOU $Y$ LESIONES CERVICALES

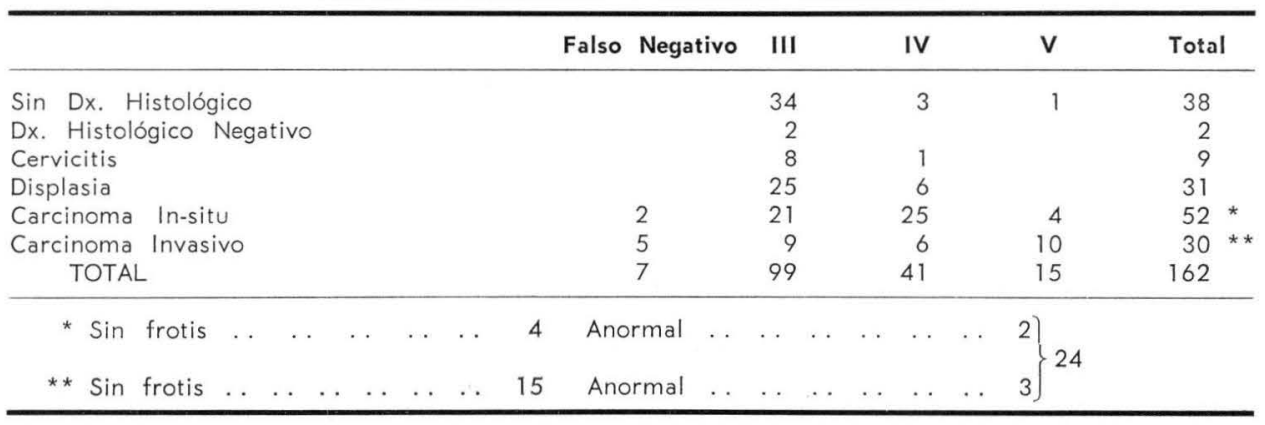

Tabla № 6. Esta Tabla muestra las diferentes clases de frotis Papanicolaou y su relación con las lesiones cervicales: 38 sin diagnóstico histológico, la mayoría de estos fueron clase 111 y en pacientes con tricomonas, quienes fueron tratados médicamente, y los frotis regresaron a la normalidad; en algunos no se obtuvo control. Entre los 186 frotis hubo 39 en los cuales se diagnosticó tricomonas, y en 7 monilia; de las 31 displasias, 11 mostraron tricomonas; de los 58 carcinoma in-situ, 9 mostraron trico- monas. Hiperplasia basocelular y displasia fueron observadas en 21 casos con tricomonas y frotis anormales, en un estudio hecho por B. Cray, W. F. Harper y R. M. Still de Stobhill General Hospital Glasgow, Scotland. (Obstetrical \& Gynecological Survey: Vol. 22, pag. 841 1967), las lesiones desaparecieron después de un tratamiento completo con Metronidazole (Flagyl); en algunos casos fueron observados cambios simulando carcinoma in-situ y algunos cambios citológicos persistieron hasta por 4 sema- 
nas después de haberse completado el tratamiento, pero nunca ha habido evidencia de relación directa causa-efecto entre tricomonas y carcinoma in-situ.

El caso con frotis clase $V$ : el paciente había sido estudiado y tratado por leucemia en el servicio médico, biopsia no se obtuvo y aparentemente no había lesión cervical visible, (reporte personal del médico tratante). Los 2 frotis clase III con histología negativa (falsos + ) se discutieron en la Tabla № 2. 9 cervicitis con frotis clase 111 . De las 31 displasias 25 tuvieron frotis clase III y 6 clase IV. Los casos de carcinoma in-situ dieron en su mayoría frotis clase IV, aunque se encontró un buen número con frotis clase III. Los frotis en carcinoma invasivo variaron $y$ fueron distribuídos casi en igual número entre las clases III, IV y V. De los 186 casos, 162 frotis fueron leídos por el departamento de citología del St. Luke's Hospital Center, y 24 fueron leídos fuera y reportados como "anormales". De los 162 frotis, 7 fueron falsos negativos (4,3\%): 2 en carcinoma in-situ y 5 en carcinoma invasivo; microfotografías de estos casos serán presentados.

En conclusión: Tricomoniasis vaginal $y / u$ otro tipo de infección cervical debe ser tratada $y$ el frotis repetido después del tratamiento, pues los cambios citológicos producidos por la infección pueden dar lugar a diagnósticos erróneos. En general, las displasias del cuello uterino dan un frotis clase III, el carcinoma in-situ: clase IV, y el carcinoma invasivo: clase $V$; sinembargo no es raro encontrar cualquiera de los frotis anormales en carcinoma in-situ o invasivo. Los frotis falso negativos se pueden explicar si la lesión es profunda en el estroma cervical sin exfoliación de células malignas.

Tabla № 7. Correlación de las biopsias múltiples con las Conizaciones: 16 pacientes fueron tratadas con conización una vez que el diagnóstico fue hecho con biopsias múltiples; se presentan los frotis pre y post-operatorio. Como puede apreciarse el diag-

TABLA N: 7

CORRELACION ENTRE LAS MULTIPLES BIOPSIAS Y LAS CONIZACIONES

\begin{tabular}{rlrcllc}
\hline & & & & & Frotis \\
post-Rx.
\end{tabular}


nóstico en ambos procedimientos corresponde bien. Las microfotografías de los casos Nos. 1, 4 y 16 se presentan: el diagnóstico de carcinoma in-situ puede pasar desapercibido en una o múltiples biopsias y éstas mostrar solamente displasia; ha sido observado por varios investigadores que cambios displásticos ocurren en la vecindad de un carcinoma in-situ, por lo tanto puede comprenderse que múltiples biopsias puedan tomar solamente el área displástica, dejando el carcinoma in-situ o aún el invasivo sin demostrar. Lo opuesto, que una simple biopsia revele carcinoma in-situ (como en los Casos Nos. 4 y 16) y la conización solamente revele displasia o cervicitis también ha si- do reportado, y probablemente representa que la lesión in-situ está apenas en su comienzo, 0 es una lesión muy pequeña, y de no ser que la lesión en el cuello sea visible, el remover una lesión in-situ con una simple biopsia no es común y puede considerarse accidental. El caso presentado como carcinoma invasivo en la biopsia y en la conización fue posteriormente tratado con radioterapia. Los frotis antes del diagnóstico histológico fueron + en 15 y falso negativo en 1 (Caso № 7) este caso será presentado en el análisis de carcinoma invasivo. En 8 casos $(50 \%)$ frotis de control fueron obtenidos, y todos regresaron negativos.

TABLA № 8

CORRELACION ENTRE LAS MULTIPLES BIOPSIAS E HISTERECTOMIAS

\begin{tabular}{|c|c|c|c|c|c|c|c|}
\hline & & & Frotis & s pre-Rx. & Biopsia & Histerectomía & $\begin{array}{c}\text { Frotis } \\
\text { post-Rx. }\end{array}$ \\
\hline 1 & DA. & $55-97-89$ & & III & Ca. Invasivo & Ca. Invasivo & $\operatorname{II}-\mathrm{R}(+)$ \\
\hline 2 & RV. & $55-45-09$ & & $\mathrm{~V}$ & & " $(R)$ & \\
\hline 3 & $\mathrm{GH}$. & $52-50-33$ & & IV & Displasia & Displasia & 1 \\
\hline 4 & WK. & $55-44-48$ & & IV & Ca. Cx./Displasia & Ca. In-situ (R) & \\
\hline 5 & RJ. & $56-51-10$ & & III & Ca. Invasivo & Ca. Invasivo (R) & 1 \\
\hline 6 & LG. & $44-99-68$ & & III & Displasia & Cervicitis & \\
\hline 7 & WL. & $53-55-59$ & & $\mathrm{v}$ & Ca. Invasivo & Ca. Invasivo (R) & 11 \\
\hline 8 & SC. & $54-96-91$ & & III & Displasia & Ca. Invasivo & I \\
\hline 9 & SC. & $51-93-86$ & & III & " & Ca. In-situ & II \\
\hline 10 & RE. & $55-52-00$ & & III & Ca. Invasivo & Cervicitis ( $R$ ) & 1 \\
\hline 11 & $\mathrm{CL}$. & $52-67-68$ & & IV & Displasia & Bowen & 11 \\
\hline 12 & WA. & $57-60-46$ & & IV & Ca. In-situ & Ca. In-situ & 11 \\
\hline 13 & so. & $57-52-74$ & & $\mathrm{~V}$ & " & Ca. Invasivo & \\
\hline 14 & RM. & $58-52-37$ & & III & Microinvasivo & $" \quad(R)$ & 1 \\
\hline 15 & WL. & $57-65-39$ & & III & Ca. In-situ & Displasia & 1 \\
\hline 16 & BA. & $58-30-11$ & & IV & Ca. Invasivo & Ca. Invasivo (R) & 1 \\
\hline 17 & VM. & $58-24-55$ & & III & " & $(R)$ & II \\
\hline 18 & McG. & $57-29-91$ & & IV & $"$ & Microinvasivo (R) & \\
\hline 19 & VMA. & $57-51-76$ & & IV & Ca. In-situ & Ca. In-situ & \\
\hline 20 & $\mathrm{GH}$. & $57-00-50$ & & III & Sospechosa & Displasia & \\
\hline 21 & AP. & $57-41-51$ & & IV & Ca. Invasivo & Ca. Invasivo (R) & $(+)$ \\
\hline 22 & McG. & $59-58-62$ & & IV & Atipia Metap. & Cervicitis Metap. & \\
\hline 23 & McL. & $57-27-20$ & & $\mathrm{v}$ & Ca. In-situ & Ca. In-situ & 1 \\
\hline 24 & LM. & $58-22-15$ & & II & Carcinosarcoma & Adenocarminoma & 1 \\
\hline 25 & $\mathrm{KJ}$. & $58-63-46$ & & normal & Atipia. Metap. & Microinvasivo & \\
\hline 26 & KG. & $43-44-39$ & & & Ca. Invasivo & Ca. Invasivo (R) & \\
\hline 27 & NE. & $56-89-32$ & & II-R & Displ. Bowenoide & " & I \\
\hline 28 & $M D$. & $57-15-24$ & & normal & Ca. In-situ & Ca. In-situ & \\
\hline
\end{tabular}


Tabla № 8. Correlación entre las múltiples biopsias e histerectomías: En 28 pacientes el diagnóstico se hizo con múltiples biopsias y fueron tratadas con histerectomía abdominal: en 18 casos el diagnóstico fue el mismo en ambos procedimientos. En 9 casos (Nos. 4, 8, 9, 10, 13, 14, 15,25 y 27) hubo disparidad en el diagnóstico; microfotografías de los casos Nos. 4, 9 y 25 se presentan; los casos Nos. 8, 10, 13, 14, 15 y 27 serán discutidos en la Tabla de carcinoma invasivo. (No fue posible conseguir fotografías de los casos 14 y 15). En este grupo hubo 10 histerectomías radicales, 8 fueron hechas por carcinoma invasivo; se practicó una histerectomía total abdominal en un caso de carcinoma invasivo, como método paliativo (Caso $\mathrm{N}$ : 1). Hubo 2 frotis falso negativos, éstos serán presentados en el análisis de carcinoma invasivo. Se obtuvieron 17 frotis de control $y$ todos regresaron negativos.

Hubo 2 muertes: una debida a carcinomatosis (Caso No 1) y la segunda debida a hemorragia incontrolable y septicemia en el 39avo. día postoperatorio (Caso N: 21 ).

De nuevo, aquí el diagnóstico de carcinoma in-situ o microinvasivo en una biopsia y carcinoma invasivo en el espécimen de la histerectomía se puede explicar con la teoría de que lesiones no invasivas se pueden encontrar adyacentes a procesos invasivos.

Tabla No 9. Correlación entre las Conizaciones y las Histerectomías: 23 casos fueron diagnosticados por medio de conización y tratados con

TABLA N: 9

CORRELACION ENTRE LAS CONIZACIONES $Y$ LAS HISTERECTOMIAS

\begin{tabular}{rlllll}
\hline & & & & & Frotis \\
post-Rx.
\end{tabular}


histerectomía; la mayoría de las histerectomías fueron hechas por carcinoma in-situ, solamente en 5 casos la lesión diagnosticada en la conización persistió en el espécimen de histerectomía: 2 displasias, 1 carcinoma in-situ, 1 microinvasivo y 1 invasivo; la explicación de esto es quizás, que la conización no se practicó lo suficientemente profunda para re- mover la lesión completamente. La efectividad de la conización es demostrada: 18 casos (fuera de 23, o $78,2 \%$ ) fueron probablemente tratados satisfactoriamente con la conización. Los frotis fueron todos positivos antes de obtenerse el diagnóstico histológico, y en 8 casos en los cuales se obtuvo frotis de control, éste fue reportado como negativo.

TABLA $N \div 10$

CONIZACIONES

\begin{tabular}{rlrccccc}
\hline & & & & & & \\
Frotis \\
post-Rx.
\end{tabular}

Tabla № 10. Conizaciones: 27 pacientes fueron tratadas con conización solamente: hubo 2 cervicitis, 13 displasias y 12 carcinomas in-situ. Los frotis fueron todos positivos con excepción de 1 (caso № 25, el cual será presentado en el análisis de carcinoma in-situ). Se obtuvieron 20 frotis de control, todos negativos. 23 pacientes fueron multíparas y 4 nulíparas. La mayoría de estos casos están bajo control ye este será de gran valor para probar la efectividad de la conización.

Tabla No 11. Correlación de biopsia, conización e histerectomía: 4 pacientes fueron diagnosticadas con 
TABLA Nํ 11

CORRELACION ENTRE LAS BIOPSIAS, CONIZACIONES E HISTERECTOMIAS

\begin{tabular}{|c|c|c|c|c|c|c|}
\hline & & & Frotis & Biopsia & Conización & Histerectomía \\
\hline 1 & CL. & $52-67-88$ & IV & Displ. Bowen & Bowen & Bowen \\
\hline 2 & PB. & $57-79-39$ & $V$ & Ca. in-situ & Ca. in-situ & Negativo \\
\hline 3 & $\mathrm{AE}$. & $57-78-54$ & III & " & " & Metaplasia \\
\hline 4 & FM. & $49-43-90$ & "Anormal" & " & " & Ca. in-situ \\
\hline
\end{tabular}

biopsia y tratadas con conización e histerectomía, todos tuvieron frotis anormales. En 2 la lesión persistió en todos 3 especímenes (casos Nos. 1 y 4); microfotografías del caso № 4 se presentan; en los otros 2 casos la lesión fue bien tratada con la conización, una paciente de 51 años y la otra de 30, ambas multíparas.

Tabla No 12. Análisis de 31 casos de Displasias Cervicales: diagnosticados y tratados con biopsia, conización y/o histerectomía. Edad: de 19 a 62 años, más prominente en la 4 a década con un promedio de 34 años, datos similares son reportados por Richart: 34,5 ; 5 pacientes fueron todos positivos con prelación de clase III.

Hubo 4 pacientes embarazadas en el momento de tomarles la biopsia y 1 en el momento de la conización. Se obtuvieron 20 frotis de control: 4 regresaron a clase $11 \mathrm{I}, 3$ de estos fueron en pacientes embarazadas: a) Caso № 3: frotis clase III durante el embarazo, la biopsia mostró displasia, un dispositivo intrauterino fue insertado en el puerperio, el frotis fue repetido dando de nuevo clase III; se practicó curetaje y biopsia cervical los cuales fueron negativos para malignidad; ésta paciente lleva 7 meses de control con frotis negativos. b) Caso № 9: frotis clase III, la conización dio displasia, los frotis de control: II-R y III; la paciente se observó por 14 meses y se perdió el control después del último frotis clase III. c) Caso № 16: frotis clase III durante embarazo, la biopsia mostró displasia, frotis de control II-R y III; la biopsia se repitió y dio cervicitit, el frotis se repitió y dio clase II; esta paciente está siendo observada y lleva 14 meses de control. d) Caso № 28: frotis clase III durante embarazo, la biopsia mostró displasia, el frotis volvió a ser clase III antes del parto; esta paciente está bajo control en el presente momento.

Estas pacientes con displasias del cuello uterino han sido observadas entre 2 y 19 meses; en 6 casos no se pudo obtener control. Hubo 2 casos en los cuales se practicó histerectomía debido a estar en duda el diagnóstico de Carcinoma in-situ en la conización (casos Nos. 12 y 13, microfotografías de estos casos se presentan).

Si queremos disminuir la incidencia de carcinoma del cuello uterino tendremos que tener un control más cuidadoso de aquellas pacientes con lesiones pre-malignas del cuello. Las 3 lesiones pre-malignas del cuello más importantes son: Hiperplasia baso-celular metaplástica, Displasia y Carcinoma in-situ; muchos investigadores creen en el lento pero constante progreso de una de estas lesiones en la siguiente y finalmente en carcinoma invasivo; en este estudio no se diagnosticaron como tales, casos de hiperplasia baso-celular. Kistner, No- 
vak, Te Linde y muchos otros advocan la teoría de que la hiperactividad baso-celular puede progresar en Carcinoma in-situ, y éste más tarde en invasivo; muchos creen que este último comienza en esta capa basal, e hiperplasia baso-celular ha sido encontrada adyacente a carcinoma in-situ e invasivo.

Según Kistner, Displasia es una forma más avanzada de hiperplasia baso-celular, envolviendo el espesor del epitelio casi en. su totalidad, con excepción de las capas más superficiales; el grado de anaplasia depende de la cantidad de hipercromatismo, del número de mitosis y de la extensión de la dispolaridad. Que algunas displasias regresan $\circ$ desaparecen espontáneamente es bien conocido, pero éstas son de un grado muy benigno, (Richart); si la displasia compromete las capas basal y parabasal más que la superficial, la lesión es más probable que progrese a una forma más avanzada en lugar de resolverse espontáneamente (Richart). Richart estudió 518 pacientes con displasia del cuello uterino, entre 0 y 900 días, 23 de éstas progresaron a Carcinoma in-situ, primordialmente entre el 181 y el 720 . día, la velocidad de progresión aumentó con la severidad de la displasia, la edad promedio fue: 34.5. (Richart R. M., Natural History of Cervical Intraepithelial neoplasia -CINClinical Obstetrics \& Gynecology, page 769, December 1967).

Ligeras displasias pueden controlarse con exámenes citológicos, tratando mientras tanto cualquier infección super-impuesta. Displasias moderadas y severas requieren múltiples biopsias o conizaciones; si la displasia continúa severa después de la conización, es aconsejable hacer histerectomía. (Harlan J. Spjut \& R. E. Fechner, from the pathology depart- ment of Baylor University, Cytological Diagnosis of Cervical Displasia \& Carcinoma In-situ, Clinical Obstetrics \& Gynecology, page 785, December 1967 ).

Diferencias en interpretación histológica entre displasia y carcinoma insitu, varían considerablemente. Un riguroso control a largo plazo y un número aceptable de casos es necesario para evaluar su curso y el mejor método es con exámenes citológicos y más recientemente con la ayuda del Colpomicroscopio. En general el frotis de las displasias, es clase III, O "lesión sospechosa".

Tabla № 13. Análisis de 58 casos de Carcinoma In-situ: Se presentan 58 casos de carcinoma in-situ, incluyendo 5 microinvasivos, los cuales fueron diagnosticados y tratados por biopsia, conización y/o histerectomía. Edades: de 23 a 61, con mayor frecuencia en la 4 a década, edad promedio: 35, aunque hubo un buen número de pacientes en la 5 a década; 8 pacientes fueron nulíparas; se encontraron 2 frotis falso negativos (casos Nos. 41 y 52 ) (las microfotografías de estos 2 casos se presentan). Dos pacientes estaban embarazadas: la primera (caso № 10) con un frotis clase IV, se practicó conización a las 8 semanas, se practicó histerectomía total abdominal y no se encontró tumor residual esta paciente se ha observado durante 15 meses. El segundo caso ( No 50) con un frotis clase III, se practicó conización a las 16 semanas, abortó 3 días más tarde; la paciente está bajo observación por su médico privado. Se obtuvieron 23 frotis de control, 1 regresó a clase III después de la conización (Caso № 15), se practicó histerectomía total abdominal encontrándose cervicitis y adenomiosis; esta paciente se ha observado durante 20 meses. 
Carcinoma In-situ fue diagnosticado en 17 casos con la conización, encontrándose negativo el espécimen de la histerectomía; en 3 casos se encontró tumor residual al hacer la histerectomía (incluyendo uno de Enfermedad de Bowen); la mayoría de las histerectomías fueron hechas entre 4 y 6 semanas después de la co. nización, con excepción de aquellas pacientes que estaban embarazadas. Se encontraron 3 casos de enfermedąd de Bowen los cuales han tenido un control de 16, 12 y 5 meses con frotis negativos, 2 fueron tratadas con histerectomía y una con conización. Hubo un caso diagnosticado con carcinoma in-situ en un pólipo (caso No 22, se presentan microfotografías), la paciente estaba tomando progestógenos, posteriormente, revisando las placas no se encontró tal carcinoma in-situ sino una hiperplasia adenomatosa de las glándulas endocervicales, para mayor seguridad se practicó un curetaje y biopsia del cuello, los cuales fueron negativos.

De los 5 casos de carcinoma microinvasivo se presentan microfotografías de los casos Nos. 54 y 57; el primero: con frotis clase III, la biopsia mostró carcinoma microinvasivo y al practicar histerectomía se encontró cervicitis. El segundo: con frotis clase IV, la biopsia mostró metaplasia escamosa atípica, sinembargo se practicó histerectomía radical pues la biopsia había sido tomada e interpretada en otro hospital como carcinoma invasivo; el espécimen de la histerectomía solo mostró displasia.

Carcinoma In-situ puede definirse así: ( según Kistner) "Anormalidad del epitelio escamoso estratificado del cuello uterino, la cual, morfológicamente se asemeja a carcinoma, pero cuya extensión es limitada a los confines del epitelio y estructuras epiteliales". Cambios morfológicos característicos: a) Indiferenciación celular con ausencia de la estratificación y maduración normal. b) Numerosas figuras de mitosis a través de la extensión del epitelio. c) Pleomorfismo celular, hipercromatismo y diskariosis. d) Ausencia de diferenciación en la superficie.

Carcinoma in-situ es probablemente un proceso irreversible y procederá a ser invasivo si no es tratado; el tiempo de transformación de in-situ a invasivo puede variar entre 11 meses y 13 años, según los diferentes datos publicados en la literatura; el problema mayor con que nos encontramos es que no se puede decir que tanto tiempo ha estado presente el proceso, en el momento en que es diagnosticado por primera vez.

De nuevo, aquí el hallazgo de un carcinoma in-situ en los márgenes de una lesión invasiva es bastante frecuente. La edad promedio reportada por Richart es 38 años. El hallazgo de algunos casos entre los 20 y 30 años de edad, hace que cada caso sea tratado individualmente, de acuerdo con la paridad, el deseo por hijos, presencia o ausencia de enfermedad pélvica.

Wespi, Younge y colaboradores, y Didle y colaboradores creen que un número pequeño de casos de carcinoma in-situ puede regresar y desaparecer espontáneamente, o después de procedimientos quirúrgicos menores, tales como una biopsia; la mayoría de estos casos así reportados han sido diagnosticados durante embarazo y han desaparecido después del parto.

Kottmeier en Estocolmo reportó un $25 \%$ de mujeres en quienes se hizo el diagnóstico de carcinoma insitu, convirtiéndose en invasivo des- 
pués de 5 años; Peterson dá datos similares: $22 \%$.

Citológicamente carcinoma in-situ es similar a una displasia severa, dominando las células basales y parabasales, siendo más numerosas las células anormales en carcinoma in-situ, éstas células redondas $u$ ovales con núcleos hipercromáticos y alargados y usualmente con citoplasma acidófilo, han sido llamadas "Células del Tercer Tipo", en contraste con las células diskarióticas de la displasia.

Ciertos puntos deben tenerse en consideración en el tratamiento de carcinoma in-situ: a) Como eventualmente el carcinoma in-situ se desarrollará en invasivo si no es tratado, la terapia es extirpación quirúrgica, principalmente histerectomía total. b) Como algunas lesiones in-situ han sido encontradas adyacentes a procesos invasivos, una histerectomía radical modificada sería el método óptimo. c) Como la lesión in-situ no se desarrolla rápidamente en invasiva, un período de observación es permisible. d) Si la paciente ha pasado la edad reproductiva $y$ ha completado sus funciones de madre, 0 tiene patología pélvica adicional, histerectomía total es generalmente practicada. e) Si la lesión es limitada a la unión escamo-columnar y el canal endocervical está normal, una conización terapéutica es aceptada.

En relación a Carcinoma Microinvasivo: en una o más áreas del epitelio hay un mínimo rompimiento de la membrana basal por células malignas. Un promedio de un $10 \%$ de carcinoma in-situ tiene microinvasión. Cuando el diagnóstico de carcinoma microinvasivo es hecho con conización, el espécimen de la histerectomía usualmente no revela la microinvasión. El tratamiento y los re- sultados son los mismos que los de Carcinoma in-situ.

Carcinoma In-situ durante Embarazo: los siguientes cambios ocurren en el cuello uterino durante el embarazo: a) Hiperplasia glandular, y b) Hiperplasia baso-celular. Algunos investigadores creen que el Carcinoma in-situ durante el embarazo, desaparece después del parto, sinembargo, Greene, Marsh y Kistner, creen que cambios específicos anaplásticos 0 neoplásticos no dependen del estímulo gestacional, y sugieren que una paciente embarazada con posible carcinoma in-situ debiera ser estudiada tan completamente como aquella que no está embarazada, especialmente para descartar invasión. El tratamiento es siempre conservativo, carcinoma in-situ no contraindica el parto por vía vaginal.

Tabla No 14. Análisis de 48 casos de Carcinoma Invasivo: 48 casos de carcinoma invasivo diagnosticados $y$ tratados con biopsia, histerectomía total abdominal, histerectomía radical, radium, y/o Co60. Edad: de 28 a 79 años, 16 casos en la 6a década, 12 en la $7^{a}, 9$ en la $5^{a}$ y 6 en la 8a. década; edad promedio: entre 55 y 60 años; Richart dá una edad promedio de 48 años. 9 pacientes fueron multíparas; hubo 5 frotis falso negativos. (Microfotografías de estos frotis se presentan). Los frotis fueron distribuídos casi igualmente entre las clases III, IV y V, sinembargo hubo prioridad de la clase $V$. Los casos fueron clasificados siguiendo la clasificación internacional, como sigue: 14 estado III, 13 estado I, 12 estado II, ( 3 estado II-a, y 9 estado II-b), 3 estado IV; 6 casos no fueron clasificados. (No se obtuvieron datos en las historias).

45 casos fueron del tipo escamoso, 2 adenocarcinomas y un carcinosar- 
coma. 5 carcinomas invasivos fueron tratados con histerectomía total abdominal; en el caso $\mathrm{N} N$. 1 , este procedimiento se hizo como paliativo después de radioterapia; en el caso № 11: la biopsia mostró displasia severa y en el espécimen de la histerectomía se encontró carcinoma invasivo, el frotis fue clase III; la paciente recibió $4.000 \mathrm{r}$ de Co60 después de la histerectomía, ha sido observada durante 19 meses con frotis normales, la clasificación se hizo en el momento de la histerectomía. Caso № 31: la biopsia mostró carcinoma in-situ, y en el espécimen de la histerectomía se encontró carcinoma invasivo; se aplicaron $2.400 \mathrm{mg}$./ hrs. de radium y $4.000 \mathrm{r}$ de $\mathrm{C} 060$ en el post-operatorio, esta paciente se ha observado durante 8 meses, con frotis normales. Caso № 39: la biopsia mostró carcinosarcoma: en el preoperatorio se aplicaron $9.100 \mathrm{mg}$./ hrs. de radium, luego se practicó histerectomía total abdominal y en el post-operatorio se aplicaron $4.000 \mathrm{r}$ de Co60; la paciente se ha observado durante 6 meses y el frotis ha sido clase I. Caso № 45: la biopsia mostró displasia Bowenoide y en el espécimen de la histerectomía se encontró invasión; adicionalmente se dieron $4.600 r$ de Co60, el frotis posttratamiento fue clase I y la paciente se ha observado por 8 meses. (Microfotografías de estos casos se presentan, Nos. 11, 31, 39, 45).

Se practicaron 11 histerectomías radicales: todos los especímenes mostraron carcinoma invasivo, con excepción del caso № 12 el cual tuvo un Adenocarcinoma en un pólipo cervical y en el espécimen de la histerectomía solo se encontró cervicitis. (Microfotografías de este caso se presentan), esta paciente ha sido observada durante 20 meses, con frotis normales. De estos pacientes trata- dos radicalmente 2 murieron: una (caso № 25) el diagnóstico se hizo en el momento de la laparotomía por fibromas, en el post-operatorio se aplicaron $2.400 \mathrm{mg} . / \mathrm{hrs}$. de radium, 4.000 r. de Co60 a la pelvis y 2.850 r. de Co60 a los linfáticos peri-aórticos; la paciente murió de carcinomatosis 12 meses después de haberse hecho el diagnóstico. La segunda muerte (caso № 36) ocurrió en el 39. día del post-operatorio, después de varios episodios de severa hemorragia vaginal y septicemia; esta muerte pudo haberse prevenido.

Otras 9 pacientes murieron con carcinoma invasivo: una durante el trabajo de estudio, sin hacerse diagnóstico histológico (caso № 4), pero clínicamente el caso era un estado IV. (Caso del servicio médico). Caso № 16: esta paciente había sido tratada por carcinoma del seno, y en la autopsia se encontró carcinoma invasivo del cuello uterino. Las otras 7 pacientes fueron tratadas con radioterapia y murieron con carcinomatosis: en 5 de éstas el diagnóstico de carcinomatosis como causa de muerte fue solamente clínico, 2 fueron confirmadas con autopsia. Finalmente hubo 3 casos de carcinoma invasivo en cuello residual.

\section{Conclusiones}

El análisis de las lesiones pre-malignas y malignas del cuello uterino en el St. Luke's Hospital Center-Woman's Hospital de Nueva York, durante los años 1966 y 1967, y la evaluación de los frotis falso-negativos ha sido presentado.

16.683 frotis cérvico-vaginales fueron teñidos siguiendo la técnica de Papanicolaou y leídos por el departamento de citología, de éstos, 186 o $1,13 \%$ fueron reportados como anormales (clases III, IV, V). Un gran 
porcentaje de frotis anormales no tuvo diagnóstico histológico y la anormalidad se creyó debida principalmente a la presencia de tricomonas; el control en muchos de estos casos se perdió y se aconseja un control más de cerca en estos casos.

El grupo más grande lo constituyó el carcinoma in-situ $(31,2 \%)$, el diagnóstico y tratamiento adecuado de estos casos, lo mismo que de las displasias del cuello uterino, ayuda a disminuir el número de casos de carcinoma invasivo.

La mayoría de los frotis anormales y lesiones cervicales fue encontrada entre los 30 y 39 años de edad; el carcinoma invasivo predominó en la 6a década de la vida. 5 displasias y 2 carcinomas in-situ fueron encontrados durante embarazo; en aquellos casos en los cuales se obtuvo control, las displasias permanecieron como tales ciespués del parto, se hará un tratamiento definitivo en estos casos; en un caso de carcinoma insito y embarazo, tratado con conización, no se encontró lesión residual 13 meses más tarde cuando fue practicada histerectomía; el efecto del embarazo en el epitelio cervical debe considerarse antes de hacer un diagnóstico cito-patológico definitivo.

En pacientes que estaban usando contraceptivos la mayoría de las lesiones observadas fueron carcinoma in-situ, predominando en aquellas pacienes bajo medicación oral. El número de casos aquí estudiados es muy pequeño para llegar a una conclusión definitiva; el efecto de los progestógenos sobre el epitelio cervical debe teners en mente antes de hacer un diagnóstico definitivo de malignidad. Un número grande de pacientes usando contraceptivos y observado por un período de tiempo adecuado es deseable.
El frotis Papanicolaou clase III fue más frecuentemente visto en displasias del cuello, teniendo como elemento principal la "célula diskariótica; el frotis clase IV se observó con predominancia en carcinoma in-situ donde la "célula del tercer tipo" es la característica.

El carcinoma invasivo dio frotis clase III, IV O V. Los frotis falso-negativos podrían explicarse si la lesión es profunda en el estroma cervical, sin producirse exfoliación de las células malignas; algunos de estos frotis falso-negativos se debieron a mala interpretación durante la lectura.

Hubo adecuada correlación entre las múltiples biopsias y las conizaciones, y la mayoría de las lesiones fueron completamente tratadas después de este último procedimiento quirúrgico, sinembargo entre las múltiples biopsias y los especímenes de histerectomía hubo considerable disparidad en el diagnóstico histológico. Esto podría ser explicado por el hecho de que lesiones pre-malignas en muchas ocasiones son encontradas adyacentes a lesiones invasivas, y éstas pueden o no ser diagnosticadas con una simple biopsia, por lo tanto haciendo que el cirujano, erróneamente, practique un procedimiento quirúrgico no indicado; sinembargo, de interés es la observación que aquellas pacientes en el presente estudio, tratadas con histrectomía total abdominal y encontrándoseles un carcinoma invasivo, y luego de radioterapia adicional están todas bien. Se aconseja que cuando el diagnóstico dado por una biopsia no es completamente claro, o hay cualquier duda en la mente del patólogo, éste procedimiento debiera ser seguido de conización, de manera que se pueda descartar invasión y establecer el tratamiento adecuado. El valor de la 
simple biopsia es solamente para probar que estamos tratando con un carcinoma invasivo. Aún conociendo que la morbilidad de la conización debe tenerse en cuenta, se ha demostrado que en buenas manos quirúrgicas, dicha morbilidad es muy limitada y en esta era avanzada de la medicina, cuando un diagnóstico histológico se puede hacer en un corto tiempo, no hay razón por la cual, una histerectomía total o radical no pueda ser hecha 48 horas después de la conización.

Un completo análisis de las displasias cervicales, carcinoma in-situ $y$ carcinoma invasivo del cuello uterino ha sido presentado, debido al relativamente pequeño número de pacientes y al corto período de control este estudio es solamente la base de un proyecto que será continuado y terminado por uno de mis colegas.

\section{BIBLIOGRAFIA}

1 ARSENAULT, J. E., AYRE y colaboradores. Obstetrics \& Gynecologic. Vol. 28 page 90, 1966.

2 FERGUSON, J. y colaboradores. Obstetrical and Gynecological Survey. Vol. 21, page 668, 1966.

3 GRAY, B., HARPER, W. F., STILL, R. M. Obstetrical \& Gynecological Survey: Vol. 22, page 841,1967

4 KISTNER, R. W. Gynecology, Principles \& Practice. Textbook, 1965.

5 NOVAK, E. R. \& WOODRUFF J. D. Gynecologic \& Obstetric Pathology. 1962.

6 PARSONS \& SOMMERS. Gynecology, Teztbook. 1962.

7 PIVER, S. y colaboradores. Obstetrics \& gynecology. Vol. 28, page 528, 1966.

8 RICHART, R. M. Clinical Obstetrics \& Gynecology, page 769. Dec. 1967.

9 SPJUT, H. J. \& FECHNER, R. E. Clinical Obstetrics \& Gynecology, page 785, Dec. 1967.

10 TE LINDE. Surgical Gynecology. Textbook). 